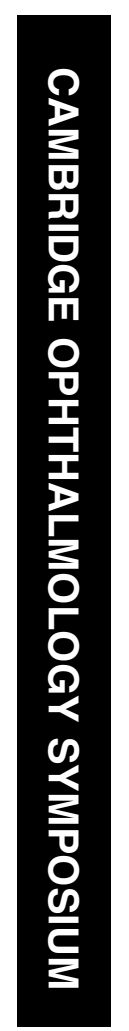

\section{Adult vitreous structure and postnatal changes}

\begin{abstract}
This review will focus on the molecular organisation of the adult vitreous and how it undergoes ageing changes throughout life that result in vitreous liquefaction and a predisposition towards posterior vitreous detachment and retinal break formation. At birth, the vitreous humour is in a gel state due to the presence of a network of fine collagen
\end{abstract} fibrils. With ageing, these collagen fibrils progressively aggregate due to a loss of type IX collagen from their surfaces. The aggregation of collagen fibrils may cause vitreous liquefaction which, when combined with an age-related weakening of postbasal vitreoretinal adhesion, predisposes to posterior vitreous detachment. Throughout postnatal life, the posterior border of the vitreous base migrates posteriorly from the ora serrata into the peripheral retina. This is due to new collagen synthesis by the peripheral retina. This new collagen intertwines with pre-existing cortical vitreous collagen to create new adhesions and thereby extends the vitreous base posteriorly. If irregularities in the posterior border of the vitreous base arise from this process, there is a predisposition towards retinal break formation during posterior vitreous detachment and subsequent rhegmatogenous retinal detachment.

Eye (2008) 22, 1214-1222; doi:10.1038/eye.2008.21; published online 29 February 2008

Keywords: vitreous; collagen; ageing; posterior vitreous detachment

\section{Introduction}

The vitreous humour is a highly hydrated tissue, with a water content of between 98-99.7\%, which is essentially composed of extracellular matrix. It is a gel-like material that in humans inevitably undergoes a process of age-related liquefaction. The vitreous humour is
MM Le Goff and PN Bishop

normally virtually acellular apart from a few cells called hyalocytes in the periphery. Hyalocytes are of bone marrow origin and are macrophage-like. ${ }^{1}$ Recently, it has been suggested that they are involved in 'vitreous cavity-associated immune deviation'. ${ }^{2}$

Despite our considerable knowledge of the composition and structure of the vitreous humour, its physiological functions remain unclear. There is evidence that it regulates eye growth and shape during development. ${ }^{3-5}$ It serves as a barrier to the cellular invasion/ migration and the diffusion of large macromolecules, this may help to maintain transparency within the vitreous cavity. ${ }^{6,7}$ Vitreous gel, as compared to liquefied vitreous, lowers oxygen tensions in the retina and lens, and the increase in lens oxygenation following vitrectomy predisposes to cataract formation. ${ }^{8}$ While its physiological roles are incompletely understood, it is clear that the vitreous and the ageing changes it undergoes are central to the pathology of several common eye conditions including rhegmatogenous retinal detachment, macular hole formation, and proliferative diabetic retinopathy.

This review will focus upon the interrelationship between vitreous biochemistry and morphology, and how biochemical changes result in ageing changes including liquefaction and posterior vitreous detachment (PVD).

Biochemical composition of the vitreous

Most extracellular matrices are composite structures containing network-forming macromolecules that possess complementary properties. There are fibrillar proteins that endow the tissue with shape, strength, flexibility, and resistance to tractional forces. Then there are charged carbohydrates, particularly glycosaminoglycans (GAGs), that attract counterions and water, thereby providing a swelling pressure that spaces apart the fibrillar proteins, inflates the tissue, and 


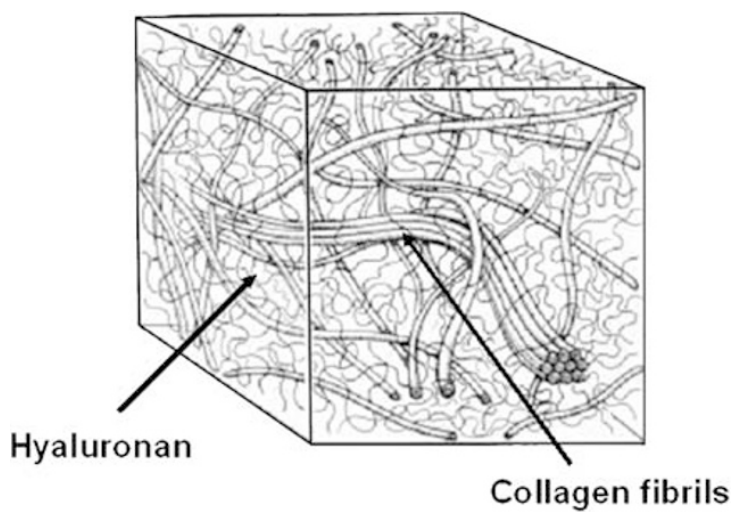

Figure 1 Schematic representation of the cooperation between two networks responsible for the gel structure of the vitreous. A network of collagen fibrils maintains the gel state and provides the vitreous with tensile strength. A network of hyaluronan fills the spaces between these collagen fibrils and provides a swelling pressure to inflate the gel.

resists compressive forces. In mammalian vitreous, the main fibrillar proteins are collagen fibrils and the major GAG is hyaluronan (HA) (Figure 1).

\section{Collagens}

The vitreous gel contains a low concentration of collagen, estimated to be approximately $60 \mu \mathrm{g} / \mathrm{ml}$ in bovine eyes and $300 \mu \mathrm{g} / \mathrm{ml}$ in human eyes. ${ }^{9}$ Balazs and Denlinger $(1982)^{10}$ showed that the total amount of vitreous collagen does not change throughout life, so the concentration decreases as the eye grows; they, therefore, suggested that there is no postnatal synthesis of vitreous collagen. However, the identification of procollagen in adult vitreous, ${ }^{11}$ the presence of immature cross-links in adult bovine vitreous, ${ }^{12}$ and morphological evidence of synthesis of vitreous collagen by the peripheral retina in the adult eye ${ }^{13}$ all suggest that some (probably low-level) postnatal collagen synthesis does occur.

Collagen molecules are formed by three polypeptide chains ( $\alpha$-chains) folding in a characteristic triple-helical configuration. This triple-helical structure requires that every third amino acid is a glycine residue, so collagen $\alpha$-chains have $(\mathrm{Gly}-\mathrm{X}-\mathrm{Y})_{n}$ amino acid repeats, where $\mathrm{X}$ and $Y$ can be any amino acids, but are frequently the imino acids proline and hydroxyproline. Glycine is the smallest amino acid, because of which it is allowed to be packed into the centre of the triple helix. The presence of hydroxyproline is important in stabilising the structure of the collagen triple helix by forming extra hydrogen bonds, while lysine and hydroxylysine residues are necessary for the formation of intramolecular cross-links, which stabilise the collagen fibril. Hydroxylysines also provide potential sites for glycosylation. All collagen molecules contain non-collagenous regions that are not folded into a triple helix. These regions are present at each end of the molecule and, in some collagens, interrupt the main triple-helical structure.

There are at least 27 different types of collagen molecules (ie collagen types I-XXVII) and these can assemble into fibrils or sheet-like structures. In the vitreous humour, nearly all of the collagen is in thin, uniform, and heterotypic (of mixed composition) fibrils containing collagen types II, IX, and V/XI. ${ }^{7,14}$ Cartilage collagen fibrils are very similar, these heterotypic fibrils contain collagen types II, IX, and XI.

\section{Type II collagen}

Collagen type II is a fibril-forming collagen that accounts for $60-75 \%$ of the collagen in vitreous. ${ }^{15}$ Molecules are composed of three identical $\alpha$-chains, that is, its composition is $\alpha 1(\mathrm{II})_{3}$. When type II collagen molecules are secreted into the extracellular environment, they are in a soluble precursor form, procollagen. Procollagen has terminal extensions called $\mathrm{N}$ - and $\mathrm{C}$-propeptide. Once within the extracellular environment, procollagen is 'processed' by specific enzymes called N-proteinase and C-proteinase (or BMP-1) that cleave the $\mathrm{N}$ - and C-propeptides, respectively, leaving small noncollagenous telopeptides at each end of the triple-helical region. This process reduces the solubility of the collagen molecules and allows them to participate in fibril formation. Processing may be incomplete leaving retained $\mathrm{N}$-propeptides on the collagen molecules.

Alternative splicing of exon 2 of the collagen type II pre-mRNA results in two forms of the $\alpha 1$ (II) chain, a long form (type IIA procollagen) and a short form (type IIB procollagen). ${ }^{16}$ The type IIA form has an additional globular domain within its N-propeptide that binds to transforming growth factor- $\beta$ and bone morphogenic protein-2. ${ }^{17}$ Fetal and adult vitreous humour contain both variants, with type IIA procollagen predominating. ${ }^{11}$ It was shown that in adult bovine vitreous, the $\mathrm{N}$-propeptides of type IIA procollagen are retained on the fibril surface. ${ }^{18}$ The relevance of this observation is not fully understood but these retained N-propeptides could bind and sequester growth factors. This differential splicing explains why patients who have been identified with mutations in exon 2 have a 'predominantly ocular' or 'ocular only' form of Stickler's syndrome. ${ }^{19}$

\section{Type IX collagen}

Type IX collagen has been estimated to represent up to $25 \%$ of the collagen in vitreous. ${ }^{14,15}$ It covalently links to the surface of heterotypic fibrils in a regular periodicity (the D-period), but it cannot form fibrils in isolation. ${ }^{20,21}$ 
It is a member of the family of fibril-associated collagens with interrupted triple helices, which also includes type XII, XIV, and XVI collagen. A common feature of members of this group is a multiple-domain structure. These are non-fibrillar collagens in which the triplehelical domains (COL domains) are separated or interrupted by short non-triple-helical domains (NC domains). Type IX collagen is a heterotrimer made of three distinct polypeptide chains, that is, its chain composition is $\alpha 1$ (IX) $\alpha 2$ (IX) $\alpha 3$ (IX) and these assemble to produce three collagenous domains (COL1, COL2, and COL3) interspersed between four non collagenous domains (NC1, NC2, NC3, and NC4). ${ }^{15,20,21}$ Further complexity is added by the $\alpha 1$ (IX) collagen gene (COL9A1) using tissue-specific alternatively spliced transcription start sites, resulting in a form in cartilage that has a globular NC4 domain, but this domain is almost absent in vitreous. ${ }^{15,22,23}$ The functional significance of these differences remains unclear.

Type IX collagen can exist in proteoglycan and nonproteoglycan forms with the proteoglycan form having a single chondroitin sulphate (CS) chain attached to the $\alpha 2(\mathrm{IX})$ chain in the NC3 domain. In vitreous, the type IX collagen is synthesised in proteoglycan form, ${ }^{15,22}$ but the length and sulphation pattern of the CS chain is species dependent. In bovine vitreous, it has a molecular weight of $15-60 \mathrm{kDa}$ and is predominantly 4 -sulphated, whereas in chick vitreous humour, the CS chain has a molecular weight of $350 \mathrm{kDa}$ and is predominantly 6-sulphated.

\section{Type V/XI collagen}

Type V/XI collagen is a minor (10-25\% of total collagen in vitreous) fibril-forming collagen that co-assembles with the type II collagen to form the core of the heterotypic fibrils. ${ }^{14}$ Type $\mathrm{V}$ collagen is found in many tissues, usually associated with type I collagen; and a variety of $\alpha$-chain combinations have been identified including $\alpha 1(\mathrm{~V})_{3,}, \alpha 1(\mathrm{~V})_{2} \alpha 2(\mathrm{~V})$, and $\alpha 1(\mathrm{~V}) \alpha 2(\mathrm{~V}) \alpha 3(\mathrm{~V})$. Type $\mathrm{XI}$ collagen is present in cartilage and has the composition $\alpha 1(\mathrm{XI}) \alpha 2(\mathrm{XI}) \alpha 3(\mathrm{XI})$, where $\alpha 1(\mathrm{XI})$ and $\alpha 2(\mathrm{XI})$ chains are distinct gene products, but $\alpha 3(\mathrm{XI})$ is the same gene product as $\alpha 1$ (II) but is more highly glycosylated Vitreous contains a hybrid molecule containing $\alpha 1(\mathrm{XI})$ and $\alpha 2(\mathrm{~V})$ chains (possibly $\alpha 1(\mathrm{XI})_{2} \alpha 2(\mathrm{~V})$, but this stoichiometry has not been confirmed); therefore, it is referred to as type $\mathrm{V} / \mathrm{XI}$ collagen. ${ }^{24}$ The $\mathrm{N}$-terminal domain of type XI collagen is not removed by processing and is retained on fibril surfaces, where it plays a key role in regulating fibril diameter by sterically hindering lateral growth of the fibril. ${ }^{25,26}$ There is evidence that collagen type $\mathrm{V}$ plays an essential role in the initiation of collagen fibril formation. ${ }^{27}$ Therefore, the type V/XI collagen may have similar roles in the context of vitreous collagen fibrils.

Linkage to the COL11A1 and COL11A2 genes encoding the $\alpha 1(\mathrm{XI})$ and $\alpha 2(\mathrm{XI})$ chains, respectively, has been established with Stickler's syndrome. ${ }^{28}$ However, Stickler's syndrome with COL11A2 mutations does not have an ocular phenotype, as the $\alpha 2(\mathrm{XI})$ chain is substituted by an $\alpha 2(\mathrm{~V})$ chain in vitreous humour.

\section{Type VI collagen}

Type VI collagen is a ubiquitous component of extracellular matrices and has been identified in small quantities in human and bovine vitreous. ${ }^{29}$ It is not a component of the heterotypic collagen fibrils but instead forms separate microfibrils. Type VI collagen has been shown to bind fibrillar collagens and $\mathrm{HA}^{30}$ so it could have a role in linking together the heterotypic collagen fibrils and HA in vitreous.

\section{Non-collagenous glycoproteins}

\section{Fibrillin}

Fibrillin-containing microfibrils have been demonstrated in vitreous by rotary shadowing electron microscopy. ${ }^{31}$ Fibrillins are a family of glycoproteins that form microfibrils with a characteristic beaded structure. Although isolated microfibrils have been isolated from vitreous, they are a minor component compared to the heterotypic collagen fibrils. However, they represent the major structural component of the zonules. ${ }^{31}$ In many tissues, fibrillin-containing microfibrils provide a scaffold for elastin assembly, but in the zonules and vitreous they are not associated with elastin. The major component of these microfibrils is fibrillin-1, this is a glycoprotein of $350 \mathrm{kDa}$ containing multiple calcium-binding epidermal growth factor (EGF)-like domains. It has been shown that the N-terminal region of fibrillin-1 binds to microfibrilassociated glycoprotein- 1 in these microfibrils. ${ }^{32}$ Marfan's syndrome is caused by mutations in fibrillin- 1 .

\section{Opticin}

Opticin is a glycoprotein that was initially identified as the major component in a pool of macromolecules extracted from the surface of vitreous (heterotypic) collagen fibrils. ${ }^{33}$ It is a member of the family of extracellular matrix small leucine-rich repeat protein/ proteoglycans. This family comprises approximately 11 members, which are characterised by a domain containing a number of tandem leucine-rich repeats (LRRs) flanked by disulphide-bonded capping motifs. LRRs are 20-30 amino-acid repeats that contain the 
consensus sequence LXXLXLXXNXL where $\mathrm{L}$ can be a leucine, isoleucine, or valine residue; $\mathrm{N}$ is an asparagine, cysteine, or threonine residue; and $X$ can be any amino acid. Opticin possesses eight tandem LRRs and an $\mathrm{N}$-terminal extension containing a cluster of sialylated O-linked oligosaccharides. Opticin exists as a dimer in solution as a result of interactions between the LRR domains. ${ }^{34}$ Recent studies have demonstrated that opticin binds growth hormone and GAGs, including heparan sulphate (HS) and CS. ${ }^{35,36}$

\section{Glycosaminoglycans and proteoglycans}

GAGs are composed of long chains of repeating disaccharide units. All GAGs except HA are attached to a protein core (thereby forming proteoglycans) and undergo varying degrees of modifications during their synthesis by sulphation, epimerisation, and acetylation/ deacetylation. Vitreous contains HA and small amounts of the sulphated GAGs, CS, and HS. ${ }^{37}$

\section{Hyaluronan}

HA is the predominant GAG in mammalian vitreous and was first isolated from bovine vitreous humour more than 70 years ago. ${ }^{38}$ It is a linear, polymeric GAG built from repeating disaccharide units $(\beta 1-4$ glucuronic acid $\beta 1-3 N$-acetylglucosamine) ${ }_{n}$. Chains of HA can be very long and form networks through entanglement. It is distinguished from the other GAGs in that it is not synthesised covalently, linked to a core protein, and it is never sulphated. HA is not uniformly distributed within the vitreous and is found in the highest concentration in the posterior vitreous cortex. ${ }^{10}$ In adult human vitreous, the HA concentration has been estimated to be between 65 and $400 \mu \mathrm{g} / \mathrm{ml}$ and the average molecular weight to be 2-4 million. ${ }^{10}$

\section{Chondroitin sulphate proteoglycans}

The repeating disaccharide unit of CS is ( $\beta 1-4$ glucuronic acid $\beta 1-3 \mathrm{~N}$-acetylgalactosamine $)_{n}$. The C-4 and/or C-6 of the $N$-acetylgalactosamine residues can be sulphated, modifying this repeating structure. The C-2 sulphation of the glucuronic acid occurs less frequently. The vitreous has been shown to contain two CS proteoglycans, type IX collagen (see section on collagens) and versican. ${ }^{15,39}$ Versican is a large proteoglycan with a central domain that carries multiple CS chains. The $\mathrm{C}$-terminal region contains EGF-like, lectin-like, and complement regulatory protein-like domains and the globular $\mathrm{N}$-terminal region contains an HA-binding domain. The binding of versican to HA is stabilised by a glycoprotein called link protein, which has also been identified in the vitreous. ${ }^{39}$ Mutations that alter the splicing of the central CS-bearing domains of versican have been implicated in the vitreoretinopathy Wagner's syndrome. ${ }^{40}$

\section{Heparan Sulphate proteoglycans}

HS and heparin share the same repeating disaccharide unit ( $\beta 1-4$ glucuronic acid $\alpha 1-4 \mathrm{~N}$-acetylglucosamine) ${ }_{n}$. This sequence can be modified by sulphation, deacetylation, and epimerisation. The degree of modification determines whether the GAG is HS or heparin. HS proteoglycans are major components of basement membranes, including the inner limiting lamina (ILL) on the inner surface of the retina. HS proteoglycans are present in the vitreous during development, but the levels are very low in postnatal eyes. $^{41}$

\section{Morphology of the vitreous}

The vitreous is subdivided into different anatomical regions (Figure 2). The central vitreous forms the bulk of the vitreous body. Within this region, the collagen fibrils generally course in an anterior-posterior direction. Anteriorly, these fibrils blend with those of the basal vitreous and posteriorly they insert into the vitreous cortex. The collagen fibrils are at their lowest concentration within the central vitreous. The collagen fibrils in the basal vitreous are at a higher concentration and are orientated perpendicular to the vitreous base, where they insert into the posterior ciliary body

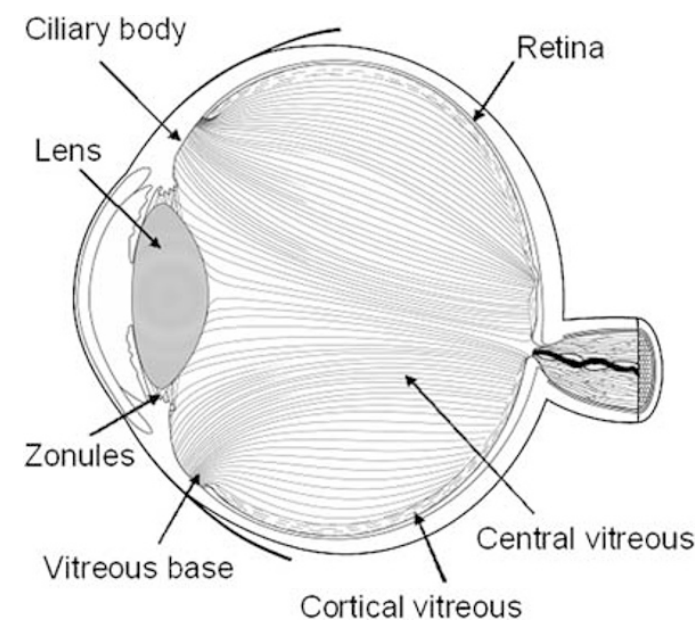

Figure 2 Diagram showing the orientation of collagen fibrils within the vitreous. Anatomically, the vitreous is subdivided into different regions including the central and cortical vitreous and the vitreous base. These regions have differing rheological properties as a result of differences in collagen fibrillar concentration and orientation. 
(pars plana) and the anterior retina, thereby forming an unbreakable adhesion. ${ }^{42}$ The vitreous cortex is a thin layer $(100-300 \mu \mathrm{m})$ that surrounds the central vitreous and has a higher concentration of collagen. The anterior cortex courses from the anterior vitreous base across to the posterior surface of the lens, and its anterior limit is called the anterior hyaloid face. This region is in direct contact with the aqueous humour and molecules can, therefore, diffuse from the vitreous into the aqueous humour. The posterior cortex lines the surface of the retina behind the vitreous base and is adherent to the ILL. There is no vitreous cortex over the optic disc and it is thinned over the macula. The cortical collagen fibrils generally are orientated parallel with the inner retinal surface and do not insert directly into the ILL. ${ }^{42-44}$ The molecular basis of vitreoretinal adhesion is poorly understood, but there may be intermediary molecules acting as a 'molecular glue' that link the cortical vitreous collagen fibrils to the ILL.

The network of (heterotypic) collagen fibrils is essential to the gel state as removal by, for example, collagenase digestion or centrifugation converts the vitreous into a viscous liquid. ${ }^{45}$ The vitreous collagen fibrils are very long, of uniform diameter $(10-20 \mathrm{~nm}$ depending upon species), and unbranched (Figure 3a). ${ }^{14}$ Analyses by freeze-etch rotary shadowing electron microscopy showed that the collagen fibrils are arranged in bundles within the vitreous and form an extended interconnected network by branching between these bundles. ${ }^{46}$ In the young vitreous, the collagen fibrils within these bundles appear to run closely together in parallel, but are not fused. Morphological analyses suggest that the CS chains of type IX collagen play a role in both connecting together and spacing apart the collagen fibrils within these bundles. ${ }^{7,47}$

\section{Ageing processes in the vitreous}

\section{PVD}

PVD is a process whereby the cortical vitreous gel splits away from ILL on the inner surface of the retina as far anteriorly as the posterior border of the vitreous base (Figure 4 ) ${ }^{48}$ It occurs in approximately $25 \%$ of the population during their lifetime. ${ }^{48,49} \mathrm{PVD}$ can be localised, partial, or 'complete' (ie up to the posterior border of the vitreous base). PVD results from a combination of vitreous liquefaction and weakening of post-basal vitreoretinal adhesion. During PVD, separation of the basal vitreous from the peripheral retina and ciliary body does not occur because of the particularly strong adhesion in this region.

While in a majority of subjects PVD occurs without major complications, in some it has sight-threatening
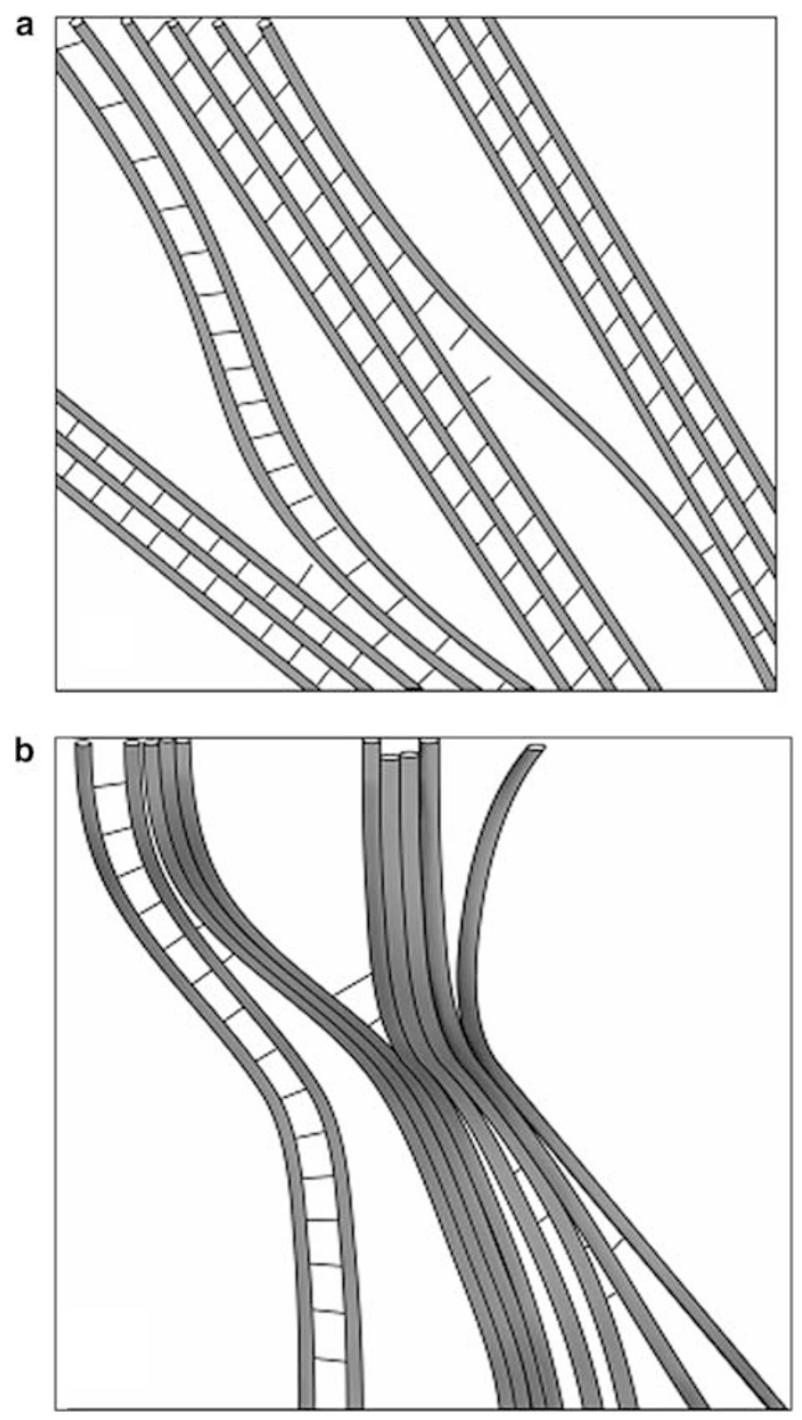

Figure 3 The collagen fibrillar network of the vitreous and ageing changes. (a) The collagen fibrils (thick grey lines) form an extended network by being organised into small bundles that are interconnected by collagen fibrils running from one bundle to another. Within each bundle, the collagen fibrils are both connected together and spaced apart by the CS chains of type IX collagen (thin black lines). (b) With ageing there is a loss of type IX collagen from the fibril surfaces. The loss of the type IX collagen CS chains from the fibril surfaces combined with an increased surface exposure of type II collagen results in collagen fibrillar aggregation.

complications. The concept of anomalous PVD was introduced by Sebag ${ }^{50}$ to describe the situation where the extent of liquefaction exceeds weakening of vitreoretinal adhesion. This results in tractional forces being exerted upon the retina during PVD and/or incomplete separation, thereby leading to complications including haemorrhage, retinal tears and detachment, macular hole formation, and vitreomacular traction syndrome. 


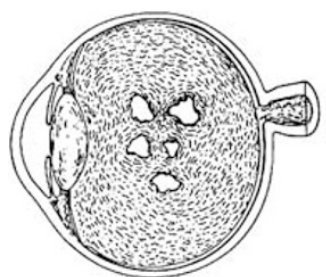

Early liquefaction

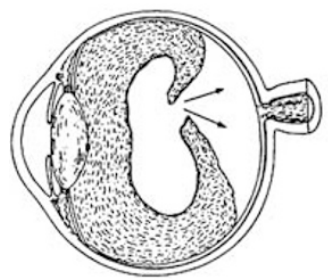

Partial PVD

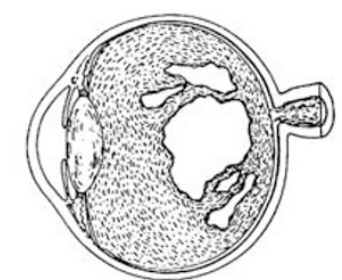

Extensive liquefaction

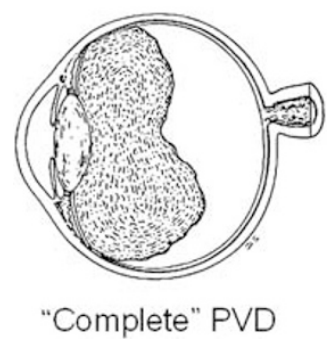

Figure 4 Age-related vitreous liquefaction and PVD. Pockets of liquid appear within the central vitreous that gradually coalesce. There is a concurrent weakening of postoral vitreoretinal adhesion. Eventually, this can progress to PVD, where the liquid vitreous dissects the residual cortical gel away from the ILL on the inner surface of the retina as far anteriorly as the posterior border of the vitreous base.

Conversely, 'complete' PVD protects against proliferative diabetic retinopathy, ${ }^{51}$ because the newly formed blood vessels require the collagenous network of the cortical vitreous as a scaffold for growth and invasion. ${ }^{52}$ In the presence of a PVD or following surgical vitrectomy, abortive neovascular outgrowths have been observed in the eyes of diabetic patients. ${ }^{51}$

\section{Vitreous liquefaction}

The human vitreous humour undergoes an inevitable process of liquefaction (or syneresis) with ageing. Studies by Balazs and Denlinger ${ }^{10}$ showed that liquid vitreous is present after the age of 4 years with around $20 \%$ of the total vitreous volume consisting of liquid vitreous by 14-18 years of age. After the age of 40 years, there is a steady increase in liquid vitreous with a concomitant decrease in gel volume. More than half of the vitreous is liquid by the age of $80-90$ years. The liquefaction process does not occur uniformly within the vitreous cavity. The pockets of liquid form in the central vitreous where they enlarge and coalesce.

Ultrastructural studies have shown that collagen fibrils aggregate with ageing into macroscopic strands within the vitreous gel. ${ }^{53,54}$ Age-related vitreous liquefaction may be caused by this gradual and progressive aggregation of the collagen fibrils, resulting in a redistribution of the collagen fibrils, leaving areas devoid of collagen fibrils and thereby converted into a liquid, and the collagen aggregates concentrated in the residual gel. ${ }^{7,47}$ An alternative hypothesis is that age-related liquefaction is a result of destruction of vitreous collagen fibrils, possibly due to enzymatic activity. ${ }^{55}$ However, recent data substantiate the former hypothesis and provide a unifying explanation for age-related vitreous liquefaction. ${ }^{47}$

Bos et $a l^{47}$ demonstrated that there is an age-related loss of type IX collagen from the surface of the heterotypic collagen fibrils of the human eye. Indeed, the half-life for the type IX collagen was found to be just 11 years of age. The CS chains of type IX collagen are thought to space the collagen fibrils apart and their loss allows the collagen fibrils to directly come into contact with one another (Figure 3b). Furthermore, the loss of type IX collagen results in the increased surface exposure of 'sticky' type II collagen so that when the type II collagen on adjacent fibrils comes into contact, there is a propensity towards fibril fusion. ${ }^{47}$ These conclusions are supported by a study showing that digestion of the vitreous with chondroitin ABC lyase, an enzyme that degrades the CS chains of type IX collagen, resulted in the aggregation of collagen fibrils. ${ }^{56}$

HA provides a swelling pressure to the collagen network and hence the vitreous gel. Complete (enzymatic) removal of the HA resulted in gel shrinkage, but not complete collapse; so HA is not a prerequisite for the gel structure. ${ }^{57}$ However, this study only looked at the short-term effects of the removal of the HA and in the longer term, it is likely to be important for the stability of the gel. It was subsequently shown that HA weakly associates with vitreous collagen fibrils and that the HA network 'stiffens' the collagenous network, as hyaluronidase digestion resulted in deflation and relaxation of the collagen network. ${ }^{46}$

\section{Weakening of the vitreoretinal adhesion}

In young eyes there is a strong adhesion between posterior vitreous cortex and the ILL of the retina; however, this weakens with ageing and this weakening is likely to be due to biochemical changes at the vitreoretinal interface. As the cortical vitreous collagen fibrils do not generally insert directly into the post-oral ILL, the basis of this adhesion may be interactions between components on the surface of the vitreous collagen fibrils and macromolecules on the inner surface of the ILL.

It has been shown that collagen type XVIII contributes towards vitreoretinal adhesion in the mouse eye, as a proportion of eyes from type XVIII collagen-knockout mice had vitreoretinal disinsertion, ${ }^{58}$ this could be due to abnormal development at the vitreoretinal interface or due to type XVIII collagen being directly involved in 


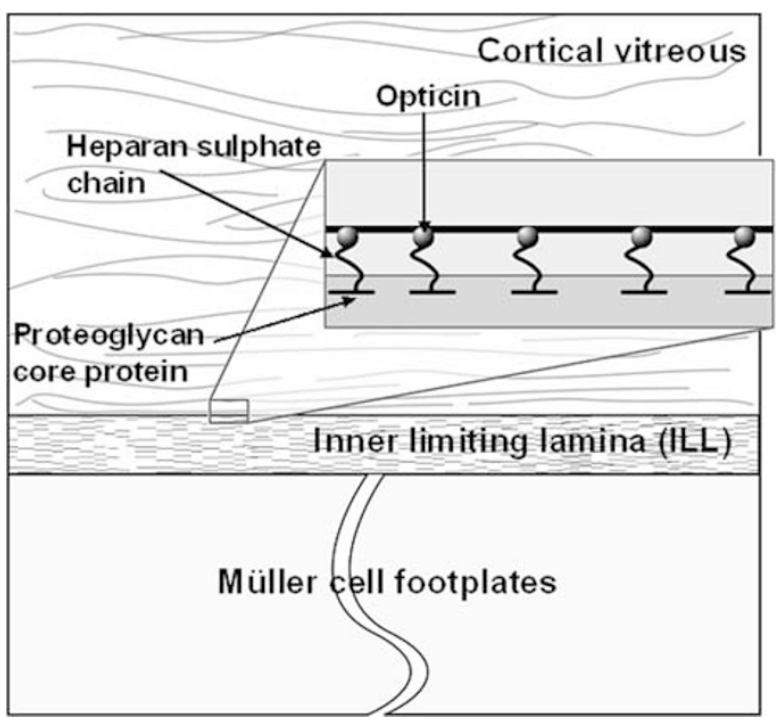

Figure 5 Diagram representing the postbasal vitreoretinal junction. Weakening of the adhesion at this interface predisposes to posterior vitreous detachment. Vitreoretinal adhesion may be dependent upon intermediary molecules acting as a 'molecular glue' and linking the cortical vitreous collagen fibrils to components of ILL. It is possible that opticin, because it binds to both vitreous collagen fibrils and HS proteoglycans in the ILL, contributes towards this 'molecular glue'.

vitreoretinal adhesion. Type XVIII collagen is an HS proteoglycan and opticin binds to HS. ${ }^{36}$ Therefore, the opticin on the surface of cortical vitreous collagen fibrils could bind HS proteoglycans of the ILL, including type XVIII collagen, thus providing a molecular basis for vitreoretinal adhesion (Figure 5). This hypothesis is supported by the colocalisation of opticin and type XVIII collagen at the vitreoretinal interface. ${ }^{59}$ However, opticinknockout mice do not appear to have spontaneous vitreoretinal disinsertion (unpublished observations), suggesting that other molecular interactions also contribute to vitreoretinal adhesion, at least in the mouse eye.

\section{Posterior extension of the vitreous base}

During PVD, the vitreoretinal separation only extends as far anteriorly as the posterior border of the vitreous base, as there is an unbreakable vitreoretinal adhesion within the vitreous base. This unbreakable adhesion is due to basal vitreous collagen fibrils running perpendicularly through defects in the ILL to merge with a network of collagen fibrils on the cellular side of the ILL or passing into 'crypts' within the cellular layers. ${ }^{13,42}$

The posterior border of the vitreous base is at the ora serrata at birth. However, it gradually migrates posteriorly as the vitreous base expands to form an annular ring that straddles the ora serrata. ${ }^{13}$ The

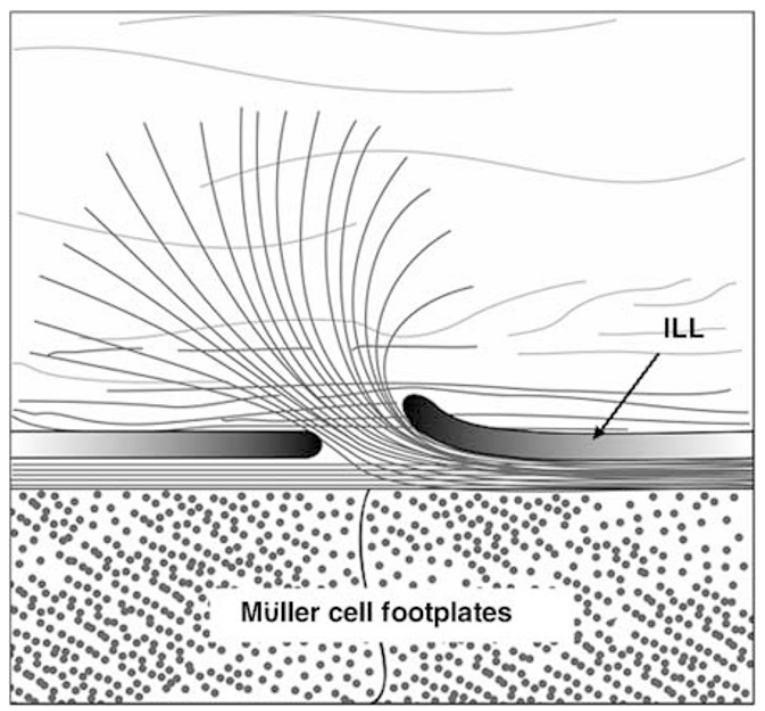

Figure 6 Diagram representing the vitreoretinal junction within the vitreous base. There is a very strong adhesion at the vitreoretinal interface within the vitreous base because vitreous collagen fibrils insert directly into the posterior ciliary body and peripheral retina. The vitreous base extends posteriorly into the peripheral retina with ageing as a result of the adult peripheral retina synthesising new collagen. This new collagen forms a layer on the cellular side of the ILL, but some breaks through defects in the ILL and intertwines with pre-existing cortical vitreous collagen thereby creating new adhesions and extending the vitreous base posteriorly.

maximum observed width of the postoral vitreous base in this study was $3.7 \mathrm{~mm}$. The extension of the vitreous base into the peripheral retina is due to synthesis of new 'vitreous' collagen by retinal cells. This collagen either forms a layer on the cellular side of the ILL or penetrates through defects in the ILL to intertwine with the preexisting cortical vitreous collagen; thereby creating new 'unbreakable' adhesions and extending the vitreous base posteriorly (Figure 6). ${ }^{13}$ If irregularities are introduced into the posterior border of the vitreous base during this process, there will be a predisposition to postoral retinal break formation and subsequent retinal detachment.

\section{Acknowledgements}

We thank Raymond Evans from the Unit of Art in Medicine at the Faculty of Medical and Human Sciences for the illustrations.

\section{References}

1 Qiao H, Hisatomi T, Sonoda KH, Kura S, Sassa Y, Kinoshita $S$ et al. The characterisation of hyalocytes: the origin, phenotype and turnover. Br J Ophthamol 2005; 89: 513-517. 
2 Sonoda KH, Sakamoto T, Qiao H, Oshima T, Tsutsumi-Miyahara C, Exley $\mathrm{M}$ et al. The analysis of systemic tolerance elicited by antigen inoculation into the vitreous cavity: vitreous cavity-associated immune deviation. Immunology 2005; 116: 390-399.

3 Coulombre AJ. The role of intraocular pressure in the development of the chicken eye. J Exp Zool 1956; 133: 211-223.

4 Arciniegas A, Amaya LE. Bio-structural model of the human eye. Ophthalmologica 1980; 180: 207-211.

5 Halfter W, Winzen U, Bishop PN, Eller A. Regulation of eye size by the retinal basement membrane and vitreous body. Invest Ophthalmol Vis Sci 2006; 47: 3586-3594.

6 Fatti I. Hydraulic flow conductivity of the vitreous gel. Invest Ophthalmol Vis Sci 1977; 16: 565-568.

7 Bishop PN. Structural macromolecules and supramolecular organisation of the vitreous gel. Prog Retin Eye Res 2000; 19: 323-344.

8 Holekamp NM, Shui YB, Beebe DC. Vitrectomy surgery increases oxygen exposure to the lens: a possible mechanism for nuclear cataract formation. Am J Ophthalmol 2005; 139: 302-310.

9 Balazs EA. Functional anatomy of the vitreus. In: Duane TD, Jaeger EA (eds). Biomedical Foundations of Ophthalmology, vol 1, Ch 17 Harper and Row: Philadelphia, 1984, pp 1-16.

10 Balazs EA, Denlinger JL. Ageing changes in the vitreous. In: Dismukes K and Sekular R (eds). Ageing and human visual function. Alan R Liss, Inc.: New York, 1982, pp 45-57.

11 Bishop PN, Reardon AJ, McLeod D, Ayad S. Identification of alternatively spliced variants of type II procollagen in vitreous. Biochem Biophys Res Commun 1994; 203: 289-295.

12 Snowden JM, Eyre DR, Swann DA. Vitreous structure. VI. Age-related changes in the thermal stability and cross-links of vitreous, articular cartilage and tendon collagen. Biochim Biophys Acta 1982; 706: 153-157.

13 Wang J, McLeod DS, Henson DB, Bishop PN. Agedependent changes in the basal retinovitreous adhesion. Invest Ophthalmol Vis Sci 2003; 44: 1793-1800.

14 Bos KJ, Holmes DF, Kadler KE, McLeod D, Morris NP, Bishop PN. Axial structure of the heterotypic collagen fibrils of vitreous humour and cartilage. J Mol Biol 2001; 306: 1011-1022.

15 Bishop PN, Crossman MV, McLeod D, Ayad S. Extraction and characterisation of the tissue forms of collagen types II and IX from bovine vitreous. Biochem J 1994; 299: 497-505.

16 Ryan MC, Sandell LJ. Differential expression of a cysteinerich domain in the amino-terminal propeptide of type II (cartilage) procollagen by alternative splicing of mRNA. J Biol Chem 1990; 265: 10334-10339.

17 Zhu Y, Oganesian A, Keene DR, Sandell LJ. Type IIA procollagen containing the cysteine-rich amino propeptide is deposited in the extracellular matrix of prechondrogenic tissue and binds to TGF-beta1 and BMP-2. J Cell Biol 1999; 144: 1069-1080.

18 Reardon A, Sandell L, Jones CJ, McLeod D, Bishop PN. Localization of pN-type IIA procollagen on adult bovine vitreous collagen fibrils. Matrix Biol 2000; 19: 169-173.

19 Richards AJ, Martin S, Yates JR, Scott JD, Baguley DM, Pope FM et al. COL2A1 exon 2 mutations: relevance to the Stickler and Wagner syndromes. Br J Ophthalmol 2000; 84: 364-371.

20 Vaughan L, Mendler M, Huber S, Bruckner P, Winterhalter KH, Irwin MI et al. D-periodic distribution of collagen type IX along cartilage fibrils. J Cell Biol 1988; 106: 991-997.
$21 \mathrm{Wu}$ J-J, Woods PE, Eyre DR. Identification of cross-linking sites in bovine cartilage type IX collagen reveals an antiparallel type II-type IX molecular relationship and type IX to type IX bonding. J Biol Chem 1992; 267: 23007-23014.

22 Yada T, Suzuki S, Kobayashi K, Kobayashi M, Hoshino T, Horie $\mathrm{K}$ et al. Occurrence in chick embryo vitreous humour of a type IX collagen proteoglycan with an extraordinarily large chondroitin sulphate chain and short $\alpha 1$ polypeptide. I Biol Chem 1990; 265: 6992-6999.

23 Brewton RG, Wright DW, Mayne R. Structural and functional comparison of type IX collagen-proteoglycan from chicken cartilage and vitreous humour. J Biol Chem 1991; 266: 4752-4757.

24 Mayne R, Brewton RG, Mayne PM, Baker JR. Isolation and characterisation of the chains of type V/type XI collagen present in bovine vitreous. J Biol Chem 1993; 268: 9381-9386.

25 Blaschke UK, Eikenberry EF, Hulmes DJS, Galla H-J, Bruckner P. Collagen XI nucleates self-assembly and limits lateral growth of cartilage fibrils. J Biol Chem 2000; 275: 10370-10378.

26 Gregory KE, Oxford JT, Chen Y, Gambee JE, Gygi SP, Aebersold $\mathrm{R}$ et al. Structural organization of distinct domains within the non-collagenous $\mathrm{N}$-terminal region of collagen type XI. J Biol Chem 2000; 275: 11498-11506.

27 Wenstrup RJ, Florer JB, Brunskill EW, Bell SM, Chervoneva I, Birk DE. Type V collagen controls the initiation of collagen fibril assembly. J Biol Chem 2004; 279: 53331-53337.

28 Brunner HG, Van Beersum SEC, Warman ML, Olsen BR, Ropers H-H, Mariman ECM. A Stickler Syndrome gene is linked to chromosome 6 near the COL11A2 gene. Hum Mol Genet 1994; 3: 1561-1564.

29 Bishop PN, Ayad S, Reardon AJ, McLeod D, Sheehan J, Kielty C. Type VI collagen is present in human and bovine vitreous. Graefe's Arch Clin Exp Ophthalmol 1996; 234: 710-713.

30 Kielty CM, Whittaker SP, Grant ME, Shuttleworth CA. Type VI collagen microfibrils: evidence for a structural association with hyaluronan. J Cell Biol 1992; 118: 979-990.

31 Ren Z-X, Brewton RG, Mayne R. An analysis by rotary shadowing of the structure of the mammalian vitreous humour and zonular apparatus. J Struct Biol 1991; 106: 57-63.

32 Cain SA, Morgan A, Sherratt MJ, Ball SG, Shuttleworth CA, Kielty CM. Proteomics analysis of fibrillin-rich microfibrils. Proteomics 2006; 6: 111-122.

33 Reardon AJ, Le Goff M, Briggs MD, McLeod D, Sheehan JK, Thornton DJ et al. Identification in vitreous and molecular cloning of opticin, a novel member of the family of leucinerich repeat proteins of the extracellular matrix. J Biol Chem 2000; 275: 2123-2129.

34 Le Goff MM, Hindson VJ, Jowitt TA, Scott PG, Bishop PN. Characterization of opticin and evidence of stable dimerization in solution. J Biol Chem 2003; 278: 45280-45287.

35 Sanders EJ, Walter MA, Parker E, Aramburi C, Harvey S. Opticin binds retinal growth hormone in the embryonic vitreous. Invest Ophthalmol Vis Sci 2003; 44: 5404-5409.

36 Hindson VJ, Gallagher JT, Halfter W, Bishop PN. Opticin binds to heparan and chondroitin sulphate proteoglycans. Invest Ophthalmol Vis Sci 2005; 46: 4417-4423.

37 Allen WS, Ottenbein E, Wardi AH. Isolation and characterisation of the sulphated glycosaminoglycans of the vitreous body. Biochim Biophys Acta 1977; 498: 167-175.

38 Meyer K, Palmer JW. The polysaccharide of vitreous humour. J Biol Chem 1934; 107: 629-634. 
39 Reardon AJ, Heinegård D, McLeod D, Sheehan JK, Bishop PN. The large chondroitin sulphate proteoglycan versican in mammalian vitreous. Matrix Biol 1998; 17: 325-333.

40 Miyamoto T, Inoue H, Sakamoto Y, Kudo E, Naito T, Mikawa $\mathrm{T}$ et al. Identification of a novel splice site mutation of the CSPG2 gene in a Japanese family with Wagner syndrome. Invest Ophthalmol Vis Sci 2005; 46: 2726-2735.

41 Halfter W, Dong S, Schurer B, Ring C, Cole GJ, Eller A. Embryonic synthesis of the inner limiting membrane and vitreous body. Invest Ophthalmol Vis Sci 2005; 46: 2202-2209.

42 Foos RY. Vitreoretinal juncture; topographical variations. Invest Ophthalmol 1972; 11: 801-808.

43 Balazs EA. Fine structure of the developing vitreous. Int Ophthalmol Clin 1975; 15: 53-63.

44 Matsumoto B, Blanks JC, Ryan SJ. Topographic variations in the rabbit and primate internal limiting membrane. Invest Ophthalmol Vis Sci 1984; 25: 71-82.

45 Balazs EA. Physiology of the vitreous body. In: Schepens CL (ed). Importance of the Vitreous Body in Retinal Surgery with Special Emphasis on Re-operation. CV Mosby Co.: St Louis, MO, 1960, pp 29-57.

46 Bos KJ, Holmes DF, Meadows RS, Kadler KE, McLeod D, Bishop PN. Collagen fibril organisation in mammalian vitreous by freeze etch/rotary shadowing electron microscopy. Micron 2001; 32: 301-306.

47 Bishop PN, Holmes DF, Kadler KE, McLeod D, Bos KJ. Age-related changes on the surface of vitreous collagen fibrils. Invest Ophthalmol Vis Sci 2004; 45: 1041-1046.

48 Foos RY, Wheeler NC. Vitreoretinal juncture: synchysis senilis and posterior vitreous detachment. Ophthalmology 1982; 12: 1502-1512.

49 Larsson L, Österlin S. Posterior vitreous detachment. A combined clinical and physicochemical study. Graefes Arch Clin Exp Ophthalmol 1985; 223: 92-95.
50 Sebag J. Anomalous posterior vitreous detachment: a unifying concept in vitreo-retinal disease. Graefes Arch Clin Exp Ophthalmol 2004; 242: 690-698.

51 Wong H-C, Sehmi KS, McLeod D. Abortive neovascular outgrowths discovered during vitrectomy for diabetic vitreous haemorrhage. Graefes Arch Clin Exp Ophthalmol 1989; 227: 237-240.

52 Faulborn J, Bowald S. Microproliferations in proliferative diabetic retinopathy and their relation to the vitreous: corresponding light and electron microscopic studies. Graefes Arch Clin Exp Ophthalmol 1985; 223: 130-138.

53 Sebag J. Age-related changes in human vitreous structure. Graefes Arch Clin Exp Ophthalmol 1987; 225: 89-93.

54 Sebag J, Balazs EA. Morphology and ultrastructure of human vitreous fibers. Invest Ophthalmol Vis Sci 1989; 30 1867-1871.

55 Los LI, van der Worp RJ, Van Luyn MJ, Hooymans JM. Age-related liquefaction of the human vitreous body: LM and TEM evaluation of the role of proteoglycans and collagen. Invest Ophthalmol Vis Sci 2003; 44: 2828-2833.

56 Goes RM, Nader HB, Porcionatto MA, Haddad A, Laicine EM. Chondroitin sulphate proteoglycans are structural renewable constituents of the rabbit vitreous body. Curr Eye Res 2005; 30: 405-413.

57 Bishop PN, McLeod D, Reardon A. Effects of hyaluronan lyase, hyaluronidase and chondroitin ABC lyase on mammalian vitreous gel. Invest Ophthalmol Vis Sci 1999; 40: 2173-2178.

58 Fukai N, Eklund L, Marneros AG, Oh SP, Keene DR, Tamarkin $\mathrm{L}$ et al. Lack of collagen XVIII/endostatin results in eye abnormalities. EMBO J 2002; 21: 1535-1544.

59 Ramesh S, Bonshek RE, Bishop PN. Immunolocalisation of opticin in the human eye. Br J Ophthalmol 2004; 88: 697-702. 